\title{
Evaluación de tipos de contenedores sobre el crecimiento radical de café (Coffea arabica L. cv. Castillo) en etapa de vivero
}

\section{Evaluation of container types on the root growth of coffee (Coffea arabica L. cv. Castillo) in the nursery stage}
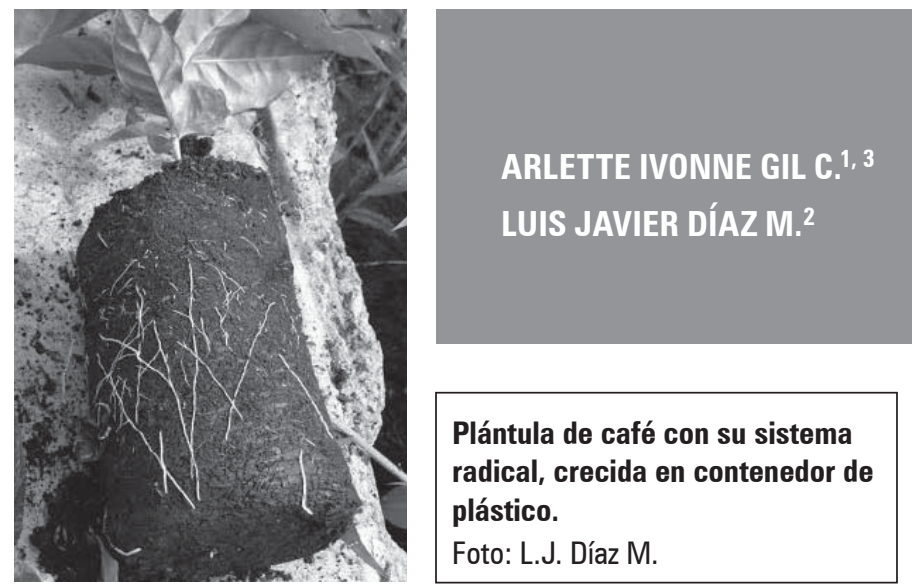

\section{RESUMEN}

Con el fin de encontrar alternativas que permitan a los caficultores producir en contenedores reutilizables plántulas más vigorosas y con un sistema radical fuerte y bien formado, se evaluó el crecimiento radical de plántulas de café, cv. Castillo, sembradas en diversos tipos de contenedores de diferentes dimensiones, determinando las variables longitud de raíz pivotante, volumen de raíces, biomasa radical y porcentaje de plantas con deformación en la raíz pivotante, en el municipio de Tibacuy (Cundinamarca). Seis tratamientos se distribuyeron en un diseño de bloques completamente aleatorizados con cuatro repeticiones, distribuidos de la siguiente manera: bandeja $3 \times 6$ (18 cavidades) de $17,3 \mathrm{~cm}$ de profundidad; bandeja $3 \times 6$ (18 cavidades) de $21,8 \mathrm{~cm}$ de profundidad; bandeja $2 \times 4$ ( 8 cavidades) de $17 \mathrm{~cm}$ de profundidad; bandeja $2 \times 4$ ( 8 cavidades) de 21,4 cm de profundidad; bolsa plástica negra de $11 \times 20 \mathrm{~cm}$ y bolsa plástica negra de $17 \times 23 \mathrm{~cm}$. Los resultados mostraron diferencias estadísticas para las variables evaluadas debido a que la restricción en el espacio de enraizamiento afectó el crecimiento radical, siendo las plantas sembradas en los contenedores de mayor profundidad las que mostraron mejores características, tales como mayor longitud de raíz pivotante, volumen de raíces y biomasa radical, destacándose aquellas sembradas en la bandeja plástica de 18 cavidades con $21,8 \mathrm{~cm}$ de profundidad, lo cual sugiere su utilización en fase de vivero, ya que debido a su característica de reutilización se puede disminuir el impacto ambiental por la utilización de bolsas de polietileno.

Programa de Ingeniería Agronómica, Grupo de investigación PROSAFIS, Universidad de Cundinamarca, Fusagasugá (Colombia).

2 Programa de Ingeniería Agronómica, Universidad de Cundinamarca, Fusagasugá (Colombia).

3 Autor para correspondencia. arlettegil@hotmail.com 


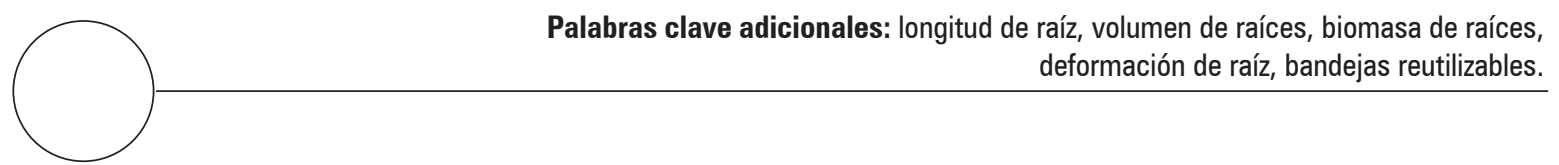

\section{ABSTRACT}

With the aim of finding alternatives that allow coffee producers to use reusable containers for more vigorous seedlings with a hard and well-formed root system, the root growth of coffee, cv. Castillo, seedlings was evaluated when sown in different types of containers with different dimensions, determining the variables of tap root length, root volume, root biomass and percentage of plants with deformation in the tap root, in the municipality of Tibacuy (Cundinamarca). Six treatments were distributed in a completely randomized block design with four repetitions, distributed as follows: $3 \times 6$ seed tray (18 wells) with a $17.3 \mathrm{~cm}$ depth; $3 \times 6$ seed tray (18 wells) with a $21.8 \mathrm{~cm}$ depth; $2 \times 4$ seed tray ( 8 wells) with a $17 \mathrm{~cm}$ depth; $2 \times 4$ seed tray ( 8 wells) with a $21.4 \mathrm{~cm}$ depth; $11 \times 20 \mathrm{~cm}$ black polyethylene bag, and $17 \times 23 \mathrm{~cm}$ black polyethylene bag. The results showed statistical differences for the evaluated variables because the restriction in the rooting space affected the root growth, with the plants sown in the containers with the greatest depth having the best characteristics, such as largest root length, root volume and root biomass. Those sown in the plastic seed tray with 18 wells and a depth of $21.8 \mathrm{~cm}$ had notable results, which recommends its use in the nursery phase due to the fact that it is reusable and that the environmental impact would be decreased.

Additional keywords: root length, root volume, root biomass, root deformation, reusable seed trays.
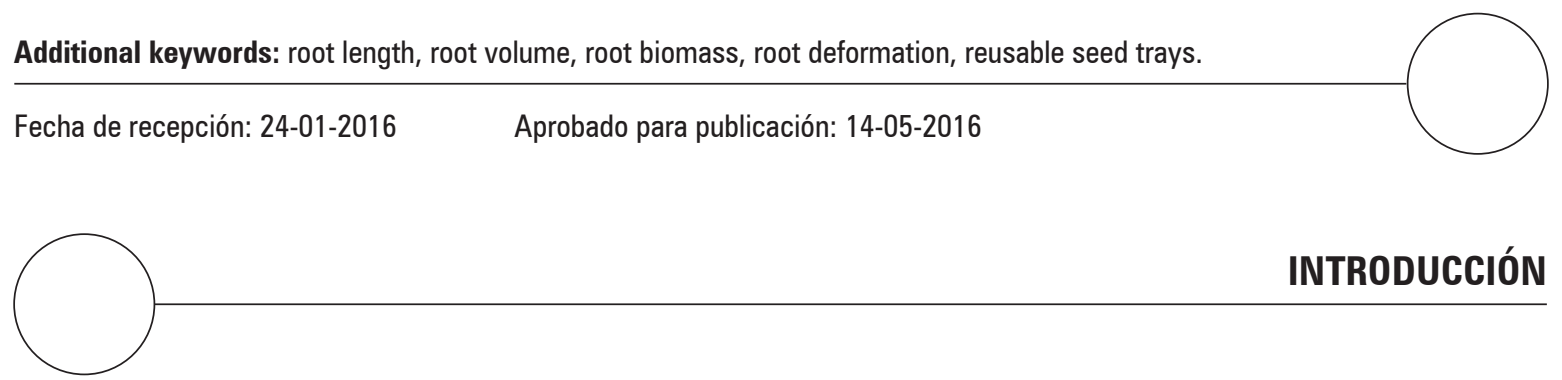

Los primeros reportes de la historia del café mencionan su origen en África oriental, lo que actualmente es Etiopía, y su domesticación se atribuye al mundo árabe (Tocancipá-Falla, 2010). La primera descripción de una planta de café fue hecha en 1592 por Próspero Alpini y un siglo después Antoine de Jussieu la denominó Jasminum arabicanum ya que la consideró un jazmín. Fue Linneo en 1737 quien la clasificó en un nuevo género, Coffea, con una sola especie conocida: $C$. arabica. Actualmente se reconocen 103 especies, sin embargo, sólo dos son responsables del 99\% del comercio mundial: Coffea arabica L. y Coffea canephora L. (Fedecafé, 2010).

El café es el cultivo agrícola de mayor importancia en la economía colombiana y se cultiva únicamente la especie C. arabica L. El Sistema de In- formación Cafetera (SICA) registra actualmente más de 564 municipios en 20 departamentos cafeteros y aproximadamente el 1\% de la superficie colombiana está cultivada con café (Gómez et al., 1991). El cultivar Castillo está compuesto de numerosas progenies de porte bajo seleccionadas en la transferencia de resistencia del híbrido de Timor a la variedad Caturra; presenta resistencia a la roya (Hemileia vastatrix Berk y Br.), es de porte bajo, con una altura media de 1,5 m y el color del cogollo de las hojas es verde a bronceado (Alvarado et al., 2005).

La cafeína (1,3,7-trimetilxantina) es un alcaloide natural producido por Coffea arabica y C. canephora, que está presente en un amplio rango de bebidas incluidas el café, el té y principalmente las bebidas gaseosas de cola. La cafeína tiene 
efectos estimulantes sobre el sistema nervioso central, junto con un rango de otros efectos positivos sobre la salud humana (Vilanova et al., 2015).

Debido a que el cafeto es una planta perenne, su explotación comercial generalmente alcanza hasta 20 años y comprende un ciclo de producción después de la siembra que dura unos seis años, con unos tres ciclos de renovación (Arcila et al., 2007). Las plantas que se consideran de buena calidad para la siembra a campo son aquellas que cuentan con un buen estado fitosanitario, óptimo desarrollo foliar y sistema radical frondoso, haciendo énfasis en la raíz principal la cual debe ser recta (Arcila, 2000).

Uno de los factores que ejerce mayor influencia sobre el crecimiento y desarrollo de las plantas en vivero es el contenedor, ya que confina las raíces en un volumen fijo de sustrato con un espacio de crecimiento limitado (Alm et al., 1982). Su principal función es la de contener una pequeña cantidad de sustrato, que a su vez abastece a las raíces con agua, aire, nutrientes minerales y además proveer soporte físico (Arizaleta y Pire, 2008). El agua en el contenedor se comporta de manera diferente que el agua en suelo no confinado (Furuta, 1978). Características como el volumen, profundidad y forma actúan sobre la dinámica de crecimiento de las raíces al limitar la disponibilidad de recursos necesarios para su crecimiento: aire, agua y nutrientes (Landis et al., 1990).

Como las plantas se encuentran confinadas en el contenedor, la cantidad de agua disponible es limitada y si la tasa de transpiración excede a la de absorción de la raíz se presenta estrés hídrico (Slatyer, 1967). Las plantas sometidas a un estrés hídrico moderado disminuyen la cantidad de energía consumida por las partes aéreas distribuyendo la mayoría de los asimilados a las raíces, incrementando su crecimiento (Ledent, 2002). Plantas con un adecuado desarrollo radical presentan más tolerancia al estrés en el trasplante y mejor comportamiento en campo (Weston y Zandstra, 1986).

El vigor relativo del sistema radical se ve reflejado en la morfología y crecimiento de la parte aérea de la planta y por esta razón muchas de las características de los contenedores fueron diseñadas para promover la relación raíz/parte aérea (Escobar, 2007). En etapa de vivero las plantas que se encuentren débiles, cloróticas, con problemas fitosanitarios y malformaciones en la raíz se deben descartar (Arcila y Botero, 1985).

Las bandejas plásticas suelen ser de poliestireno expandido o espuma flex y están disponibles en una variedad de tamaños y formas de cavidad, siendo extremadamente durables ya que tienen una expectativa de vida de más de 10 años (Dumroese et al., 2009). Las celdas son cónicas, de diámetro y longitud variable y cuentan con orificio de drenaje en su base (Allison, 1974).

El Centro Nacional de Investigaciones de Café (Cenicafé) recomienda el uso de bolsas plásticas de $2 \mathrm{~kg}$ de capacidad $(17 \mathrm{~cm}$ de diámetro por 23 $\mathrm{cm}$ de altura) para un período de hasta 6 meses, pero debido a diversos factores, en la producción comercial la permanencia de la planta en bolsa es de cuatro meses, por lo que se usan de menor capacidad (Salazar-Arias, 1991). La bolsas se rompen durante el trasplante por lo que no son reutilizables, lo cual aumenta los costos de producción (Serrada, 2000). Al usar un contenedor más pequeño el productor pretende reducir los costos de producción, aprovechar mejor el espacio y transportar las plantas fácilmente, pero esto puede afectar su calidad (Salazar-Arias, 1996).

De acuerdo con lo anterior, esta investigación fue realizada con el fin de evaluar la influencia del tipo de contenedor sobre el crecimiento de plantas de café en etapa de vivero, mediante diversos parámetros de raíz como longitud de la raíz pivotante, volumen de raíces, biomasa radical y porcentaje de plantas con deformaciones, 
para obtener plantas más vigorosas, con un sistema radical fuerte y bien formado, en contenedores reutilizables, disminuyendo los costos de producción y el impacto ambiental debido al excesivo uso de bolsas plásticas.

\section{MATERIALES Y MÉTODOS}

Para el desarrollo de esta investigación, primero se realizó una fase de campo y posteriormente una de laboratorio. La fase de campo se llevó a cabo en la granja Alberto J. Williamson del Comité de Cafeteros de Cundinamarca, vereda La Portada, municipio de Tibacuy (Cundinamarca), sobre las coordenadas $4^{\circ} 22^{\prime} \mathrm{N}$ y $74^{\circ} 26^{\prime} \mathrm{W}$, a una altitud de 1.538 msnm, temperatura media de $19,2^{\circ} \mathrm{C}$, humedad relativa de $78,1 \%$ y precipitación promedio anual de $1.096 \mathrm{~mm}$. La fase de laboratorio se realizó en las instalaciones de la Universidad de Cundinamarca, sede Fusagasugá, en el laboratorio de Fisiología Vegetal.

Se implementó un diseño de bloques completos al azar (BCA) con seis tratamientos y cuatro repeticiones, para un total de 24 unidades experimentales. Se evaluaron los siguientes tratamientos: bandeja de 18 cavidades $(3 \times 6)$ con $17,3 \mathrm{~cm}$ de profundidad (tratamiento 1), bandeja de 18 cavidades $(3 \times 6)$ con $21,8 \mathrm{~cm}$ de profundidad (tratamiento 2), bandeja de 8 cavidades $(2 \times 4)$ con 17 $\mathrm{cm}$ de profundidad (tratamiento 3 ), bandeja de 8 cavidades $(2 \times 4)$ con $21,4 \mathrm{~cm}$ de profundidad (tratamiento 4), bolsa de polietileno negro de $11 \times 20$ $\mathrm{cm}$ (tratamiento 5) y bolsa de polietileno negro de $17 \times 23 \mathrm{~cm}$ (tratamiento 6) (tabla 1).

El ensayo se desarrolló en la sección 4 de la zona de viveros de la granja, la cual contó con tres camas de $10 \mathrm{~m}$ de longitud por 1,2 $\mathrm{m}$ de ancho. Las plantas se ubicaron en las camas 1,2 y 3 , en lotes de 100 plantas. Se utilizó malla polisombra del $50 \%$ para cubrir el área de la zona de viveros con el fin de proteger a las plántulas del exceso de radiación solar. Se colocaron un total de 24 lotes correspondientes a la totalidad del ensayo.
Como material vegetal se utilizaron plántulas de café (C. arabica L. cv. Castillo) de $60 \mathrm{~d}$ de edad. El sustrato utilizado para llenar los contenedores fue suelo cernido, cascarilla de arroz y gallinaza en proporción 3:1:1. El suelo utilizado poseía textura franco-arcillosa, $\mathrm{pH}$ ligeramente ácido $(6,5)$, contenido medio de materia orgánica $(2,7 \%)$ y baja conductividad eléctrica $(0,27$ $\left.\mathrm{dS} \mathrm{m}^{-1}\right)$. La siembra se realizó con un punzón de madera, teniendo cuidado que la raíz quedara recta y se mantuviera firme. La fertilización dada a las plántulas fue únicamente edáfica, con dosificación de $0,40 \mathrm{~g} /$ planta de $\mathrm{N}, 0,87 \mathrm{~g} /$ planta de $\mathrm{P}_{2} \mathrm{O}_{5}$ y $0,08 \mathrm{~g} /$ planta de $\mathrm{K}_{2} \mathrm{O}$. Se aplicó riego por aspersión según las necesidades del cultivo, además de las labores propias de la granja para manejo de malezas, plagas y enfermedades.

Los muestreos se realizaron semanalmente comenzando a los $7 \mathrm{~d}$ después del trasplante (ddt) y finalizando a los $112 \mathrm{ddt}$. El tamaño de la muestra fue de cuatro plantas tomadas al azar dentro de las unidades experimentales. Las muestras se tomaron de las 8:00 a 9:00 h, con el fin de evitar la deshidratación del material vegetal. Las plántulas seleccionadas se retiraban del contenedor y se limpiaban sus raíces con cuidado hasta remover la totalidad del sustrato presente. Una vez limpias se introdujeron en bolsas de papel rotuladas y fueron colocadas en contenedor de icopor para ser llevadas al laboratorio de manera inmediata.

Las variables evaluadas fueron: longitud de la raíz pivotante (en $\mathrm{cm}$, realizada con calibrador), volumen de raíces (en $\mathrm{cm}^{3}$, basado en el principio de Arquímedes de desplazamiento de volumen), biomasa radical (en g, determinada en estufa de secado a $80^{\circ} \mathrm{C}$ hasta peso constante) y porcentaje de plantas (\%) con deformación de raíz pivotante (relación entre número de plantas con malformaciones morfológicas de la raíz y el total de plantas muestreadas). El análisis estadístico de los datos fue realizado en el programa SAS versión 9.0 para análisis de varianza y pruebas de comparación múltiple de Tukey con un grado de significancia $(\alpha)$ de 0,01 . 
Tabla 1. Tratamientos establecidos en la evaluación de tipos de contenedores sobre el crecimiento radical de café (C. arabica L. cv. Castillo) en etapa de vivero.

\begin{tabular}{|c|l|}
\hline Tratamiento & \multicolumn{1}{|c|}{ Tipo contenedor } \\
\hline 1 & $\begin{array}{l}\text { Bandeja plástica 3x6 (18 cavidades) de 17,3 } \\
\text { cm de profundidad (referencia A-BA18SP) }\end{array}$ \\
\hline 2 & $\begin{array}{l}\text { Bandeja plástica 3x6 (18 cavidades) de 21,8 } \\
\text { cm de profundidad (referencia A-BA18UP) }\end{array}$ \\
\hline 3 & $\begin{array}{l}\text { Bandeja plástica 2x4 (8 cavidades) de 17 cm } \\
\text { de profundidad (referencia A-BA8P) }\end{array}$ \\
\hline 4 & $\begin{array}{l}\text { Bandeja plástica 2x4 (8 cavidades) de 21,4 cm } \\
\text { de profundidad (referencia A-BA8SP) }\end{array}$ \\
\hline 5 & $\begin{array}{l}\text { Bolsa polietileno negro de 11x20 cm } \\
\text { (volumen de } 750 \mathrm{~cm}^{3} \text { ) }\end{array}$ \\
\hline 6 & $\begin{array}{l}\text { Bolsa polietileno negro de } 17 \times 23 \mathrm{~cm} \\
\text { (volumen de } 1.700 \mathrm{~cm}^{3} \text { ) }\end{array}$ \\
\hline
\end{tabular}

\section{RESULTADOS Y DISCUSIÓN}

\section{Longitud de la raíz pivotante}

De acuerdo con los resultados obtenidos para esta variable, al final del ensayo (112 ddt) se presentaron diferencias estadísticas $(P \leq 0,01)$ entre tratamientos, teniendo los mayores valores para las plántulas sembradas en bandeja de $21,8 \mathrm{~cm}$ de profundidad y la bolsa de $17 \times 23 \mathrm{~cm}$, con 19,75 y $19 \mathrm{~cm}$, respectivamente. Los menores valores se presentaron para aquellas sembradas en bolsa de $11 \times 20 \mathrm{~cm}$ con $15,81 \mathrm{~cm}$ y bandeja de $17,3 \mathrm{~cm}$ de profundidad con $15,12 \mathrm{~cm}$ de raíz, como se observa en la tabla 2.

La elongación de la raíz es un proceso continuo que es esencial para el desarrollo de las plantas, que se ve influenciado por las características del medio en el cual se encuentra como la disponibilidad de aire, agua y nutrientes, entre otros (Kafkafi, 2008). Cuando las plantas son producidas en diferentes tipos de contenedores, el crecimiento generalmente está influenciado por el volumen que el contenedor posee para el desarrollo de la raíz (Bilderback, 2001). Todos los contenedores crean una franja húmeda de sustrato y la profundidad de esta capa saturada está en función de la altura del contenedor y de las propiedades físicas del sustrato (Wallach y Raviv, 2005).

Las plántulas sembradas en contenedores de menor volumen y profundidad presentaron disminución en el desarrollo de la raíz pivotante debido a la limitación de diversos factores, tal como lo corrobora Spomer (1982), debido a que la distribución del volumen del contenedor es de gran importancia en plantas con raíz pivotante fuerte: dos plantas sembradas en contenedores con el mismo volumen pueden mostrar diferencias en el crecimiento de la raíz si la profundidad es diferente (Berenguer et al., 2004). Las raíces son muy sensibles a las condiciones estresantes del suelo, enviando señales hacia la parte aérea, las cuales pueden afectar la conductancia estomática, la expansión celular, la división celular y la tasa de aparición foliar (Passioura, 2002).

Seis acuaporinas homólogas fueron identificadas en tres especies de café (Coffea arabica, C. canephora y C. racemosa), incluidas cuatro proteínas intrínsecas de membrana (PIP2;1, PIP1;2, PIP2;1 y PIP2;2). La expresión de PIP2;1 y PIP2;2 fue evidentemente regulada en hojas y raíces debido al déficit hídrico, sugiriendo el posible rol de estos genes para controlar el estatus hídrico de las plantas y en la recuperación posestrés de plantas irrigadas (Brombini y Mazzafera, 2013).

\section{Volumen de raíces}

Los resultados mostraron que las plántulas de café respondieron a la limitación en el espacio de siembra de acuerdo con su volumen de raíces, presentando mayor desarrollo las que fueron sembradas en contenedores de mayor capacidad. De acuerdo con ello, a los 112 ddt hubo diferencias estadísticas $(P \leq 0,01)$ entre tratamientos, para el volumen de las raíces, teniendo que aquellas sembradas en bandejas de $21,4 \mathrm{~cm}$ y $21,8 \mathrm{~cm}$ de profundidad obtuvieron 6,26 y $6,21 \mathrm{~cm}^{3}$, respectivamente (tabla 2). En contraste, el menor volumen se presentó en las ubicadas en bolsa de 
Tabla 2. Efecto del tipo del contenedor sobre la longitud y volumen de raíces de café (C. arabica L. cv. Castillo) en etapa de vivero, a los $112 \mathrm{ddt}$.

\begin{tabular}{|c|c|c|c|}
\hline Tratamiento & Tipo contenedor & $\begin{array}{l}\text { Longitud de raíz pivotante } \\
\qquad(\mathrm{cm})\end{array}$ & $\begin{array}{l}\text { Volumen de raíces } \\
\qquad\left(\mathrm{cm}^{3}\right)\end{array}$ \\
\hline 1 & $\begin{array}{l}\text { Bandeja plástica } 3 \times 6 \\
(17,3 \mathrm{~cm} \text { de profundidad) }\end{array}$ & $15,12 \mathrm{c}$ & $4,52 b$ \\
\hline 2 & $\begin{array}{l}\text { Bandeja plástica } 3 \times 6 \\
(21,8 \mathrm{~cm} \text { de profundidad) }\end{array}$ & 19,75 a & $6,21 \mathrm{a}$ \\
\hline 3 & $\begin{array}{l}\text { Bandeja plástica } 2 \times 4 \\
\text { (17 cm de profundidad) }\end{array}$ & $16,27 \mathrm{bc}$ & $5,78 a b$ \\
\hline 4 & $\begin{array}{l}\text { Bandeja plástica } 2 \times 4 \\
\text { (21,4 cm de profundidad) }\end{array}$ & $18,77 \mathrm{ab}$ & 6,26 a \\
\hline 5 & $\begin{array}{l}\text { Bolsa polietileno negro de } 11 \times 20 \mathrm{~cm} \\
\text { (volumen de } 750 \mathrm{~cm}^{3} \text { ) }\end{array}$ & $15,81 \mathrm{c}$ & $4,63 \mathrm{~b}$ \\
\hline 6 & $\begin{array}{l}\text { Bolsa polietileno negro de } 17 \times 23 \mathrm{~cm} \\
\text { (volumen de } 1.700 \mathrm{~cm}^{3} \text { ) }\end{array}$ & 19,00 a & $5,33 a b$ \\
\hline
\end{tabular}

Promedios con letras distintas indican diferencia significativa según la prueba Tukey $(P \leq 0,01)$.

polietileno de $11 \times 20 \mathrm{~cm}$ con $4,63 \mathrm{~cm}^{3}$ y bandeja de $17,3 \mathrm{~cm}$ de profundidad con un valor de 4,52 $\mathrm{cm}^{3}$. La figura 1 muestra el volumen de raíces en café, de acuerdo con cada tratamiento evaluado.

Estudios similares realizados en café (C. arabica L.) (Arizaleta y Pire, 2008), cacao (Theobroma cacao L.) (Gutiérrez et al., 2011), eucalipto (Eucalyptus viminalisLabill.) (Al-Zalzaleh, 2009), limón (Citrus limon (L.) Burm.) (Ouma, 2006) y pino (Pinus palustris) (South et al., 2005), donde se evaluó la limitación del volumen del suelo como origen de restricción radicular, indicaron que las plantas desarrolladas en contenedores pequeños presentaron restricción en el crecimiento radical, ya que la raíz en crecimiento representa el principal vertedero metabólico de fotoasimilados y al limitar su espacio de crecimiento se reduce su fortaleza como vertedero, generando un desequilibrio en la relación fuente-vertedero (Barrett y Gifford, 1995). Zaharah y Razi (2009) encontraron que la restricción de las raíces en mango (Mangifera indica cv. Chokanan) contribuyó a la reducción foliar, inhibiendo así el crecimiento de los árboles.

Un suelo compactado presenta reducción en la cantidad de macroporos e incremento en su densidad, lo que resulta en menor infiltración de agua, además de desfavorecer la profundidad del sistema radical de las plantas, con resultados perjudiciales para su crecimiento, principalmente en situaciones de déficit hídrico (Pereira et al., 2015).

\section{Biomasa seca radical}

Al finalizar el ensayo (112 ddt) los resultados mostraron que las plántulas sembradas en bandejas plásticas de 21,4 cm y 21,8 cm de profundidad acumularon la mayor cantidad $(P \leq 0,01)$ de materia seca en las raíces, con 0,82 y $0,81 \mathrm{~g}$, respectivamente, mientras que las ubicadas en bolsa de polietileno de $11 \times 20 \mathrm{~cm}$ y en bandeja plástica de $17,3 \mathrm{~cm}$ de profundidad obtuvieron valores de 0,61 y $0,60 \mathrm{~g}$ respectivamente (tabla 3 ).

Estos resultados están de acuerdo con trabajos realizados por Coopman (2000), quien afirma que en plantas producidas en contenedores, la biomasa del sistema radical está directamente relacionada con la profundidad, volumen y forma del contenedor. En general, tanto más grande sea el contenedor, más grande será la planta que puede ser producida en él (Kinghorn, 1974). 


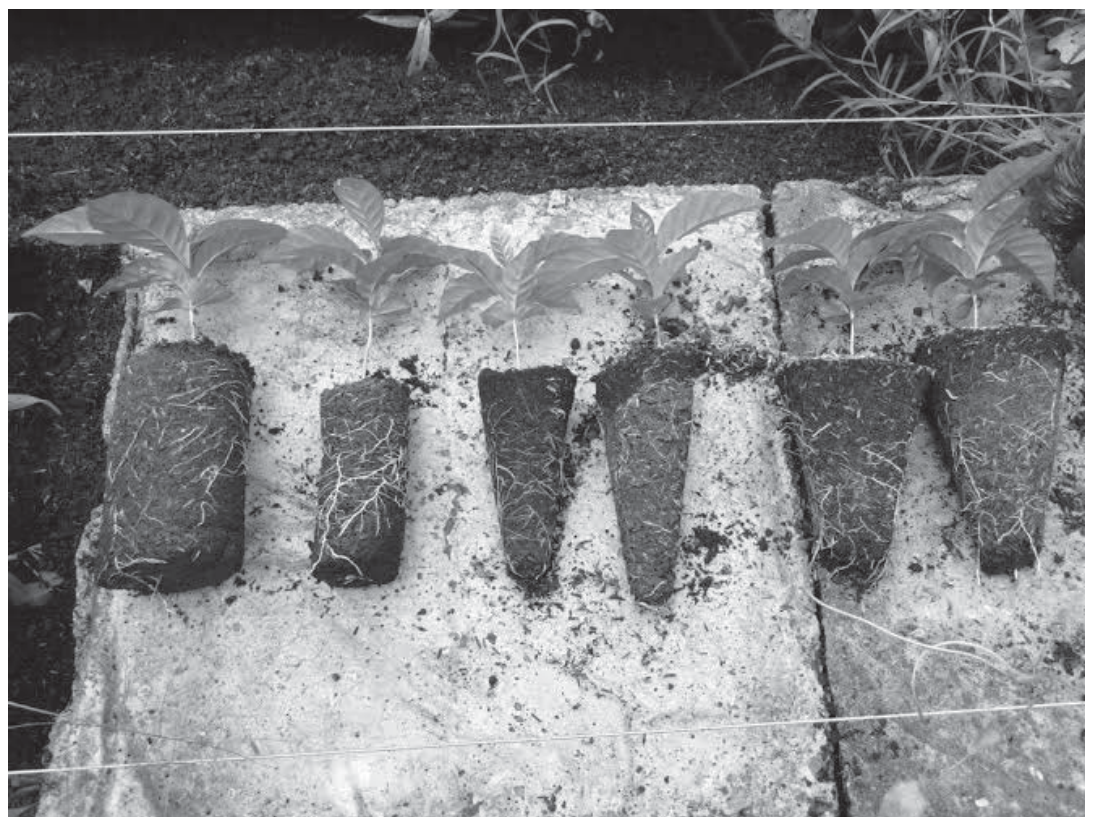

Figura 1. Volumen de raíces en café ( $C$. arabica L. cv. Castillo) en etapa de vivero, a los 112 ddt. De izquierda a derecha se muestran plántulas sembradas en: bolsa de polietileno negro de 17x23 (tratamiento 6), bolsa de polietileno negro de 11×20 cm (tratamiento 5), bandeja plástica $3 \times 6$ de 17,3 cm de profundidad (tratamiento 1), bandeja plástica $3 \times 6$ de $21,8 \mathrm{~cm}$ de profundidad (tratamiento 2), bandeja plástica $2 \times 4$ de $17 \mathrm{~cm}$ de profundidad (tratamiento 3 ) y bandeja plástica $2 \times 4$ de $21,4 \mathrm{~cm}$ de profundidad (tratamiento 4). Foto: L.J. Díaz M.

Cuando las raíces están confinadas en contenedores que restringen su crecimiento, se incrementa la competencia por recursos esenciales (Peterson et al., 1991a); el incremento en el tamaño de la raíz y la disminución en el espacio de enraizamiento generan competencia por el oxígeno disponible (Peterson et al., 1991b). Al disminuir la profundidad y el volumen del contenedor, la cantidad del espacio poroso disminuye consecuentemente reduciendo la capacidad de retención de agua y aire (Bilderback y Fonteno, 1991). Plantas de ají (Capsicum annum L.) sembradas en contenedores más pequeños indujeron floración temprana de corta duración, lo que conllevó a una maduración precoz no deseable debido a una mayor competencia por macronutrientes (Xu et al., 2001).

El contenido de nitrato endógeno en las raíces se ve severamente reducido bajo condiciones de hipoxia, indicando su movilización. Aunque su transporte a la parte aérea vía xilema se reduce bajo déficit de oxígeno, la severidad de esta disminución depende de la concentración de nitrato en el medio, sugiriendo que al menos algo del nitrato en el xilema proviene del medio. La nitrato-reductasa de las raíces se ve fuertemente reducida bajo hipoxia, pero se recobra rápidamente cuando se vuelve a la normoxia (Brandão y Sodek, 2009).

Los regímenes de irradiancia influencian la actividad de la nitrato-reductasa y su partición entre hojas y raíces en plántulas de café (C. arabica L.). La actividad de la enzima en hojas tuvo correlaciones positivas con la asimilación del $\mathrm{CO}_{2}$, en respuesta a los regímenes de irradiancia. Bajo condiciones favorables de asimilación de $\mathrm{CO}_{2}$, mayor actividad de la nitrato-reductasa fue observada tanto en hojas como en raíces, y una 
tendencia contraria ocurrió bajo condiciones limitantes de $\mathrm{CO}_{2}$. Bajo régimen moderado de irradiancia las hojas fueron el principal sitio de reducción del nitrato, contribuyendo con el 70\% del total de la asimilación del nitrato en la planta (Carelli y Fahl, 2006).

\section{Porcentaje de plantas con deformación en la raíz pivotante}

Los resultados a los $112 \mathrm{ddt}$ presentaron diferencias estadísticas $(P \leq 0,01)$ entre tratamientos, mostrando que las plántulas sembradas en bolsa de polietileno de $17 \times 23 \mathrm{~cm}$ obtuvieron el mayor porcentaje de deformación en la raíz pivotante con un 39,06\%, en contraste con las ubicadas en bandejas plásticas de 21,8 y 21,4 cm de profundidad, que presentaron los menores valores para esta variable con un 15,63 y $19,93 \%$ respectivamente (tabla 3 ). En la figura 2 se observa la deformación en la raíz pivotante, generando el enrollado de la misma.

Las raíces de las plantas crecen geotrópicamente, pero si encuentran un obstáculo físico al llegar al fondo del contenedor tienden a crecer lateralmente sobre la superficie interna (Burdett, 1979). Para las plántulas de café sembradas en bolsas, la raíz pivotante suele doblarse y crecer de nuevo hacia arriba o enrollarse en el fondo del contenedor, coincidiendo con lo descrito por Ball (1976) en la producción de plántulas de pino (Pinus sp.) en bolsa plástica. Los contenedores usados para café deben promover un desarrollo adecuado de la raíz, impidiendo que se doble en el fondo generando "enrollado" al final del envase (Wightman, 2000). Este tipo de deformación genera la denominada "cola de marrano" en las plantas adultas en campo, causando inestabilidad física de las plantas y pérdida de capacidad de absorción de nutrientes (Gaitán et al., 2011). Plántulas de Quercus suber L. sembradas en contenedores más profundos presentaron una mayor longitud de la raíz principal, sin deformaciones, con mayor biomasa radical y aérea (Chirino et al., 2008).

Una apropiada profundidad y forma geométrica del contenedor es esencial para obtener una adecuada disponibilidad de agua en la zona radical de las plantas sembradas en estos (Heller et al., 2015) y poder entender los procesos que tienen lugar dentro del sistema continuo raíz-planta-atmósfera, así como las interacciones entre el sustrato de crecimiento, las fases líquida y gaseosa dentro de esta matriz y su estatus nutricional (Raviv et al., 2008).

Tabla 3. Efecto del tipo del contenedor sobre la biomasa seca radical y porcentaje de plantas con deformación en la raíz pivotante de café (C. arabica L. cv. Castillo) en etapa de vivero, a los 112 ddt.

\begin{tabular}{|c|l|c|c|}
\hline Tratamiento & \multicolumn{1}{|c|}{ Tipo contenedor } & $\begin{array}{c}\text { Biomasa seca radical } \\
(\mathrm{g})\end{array}$ & $\begin{array}{c}\text { Porcentaje de plantas con } \\
\text { deformación en la raíz pivotante }\end{array}$ \\
\hline 1 & $\begin{array}{l}\text { Bandeja plástica 3x6 } \\
(17,3 \mathrm{~cm} \text { de profundidad) }\end{array}$ & $0,60 \mathrm{~b}, 35 \mathrm{~b}$ \\
\hline 2 & $\begin{array}{l}\text { Bandeja plástica } 3 \times 6 \\
(21,8 \mathrm{~cm} \text { de profundidad) }\end{array}$ & $0,81 \mathrm{a}$ & $15,63 \mathrm{a}$ \\
\hline 3 & $\begin{array}{l}\text { Bandeja plástica } 2 \times 4 \\
(17 \text { cm de profundidad) }\end{array}$ & $0,76 \mathrm{ab}$ & $29,69 \mathrm{~b}$ \\
\hline 4 & $\begin{array}{l}\text { Bandeja plástica } 2 \times 4 \\
(21,4 \text { cm de profundidad) }\end{array}$ & $0,82 \mathrm{a}$ & $19,93 \mathrm{a}$ \\
\hline 5 & $\begin{array}{l}\text { Bolsa polietileno negro de } 11 \times 20 \\
\mathrm{~cm}\left(\text { volumen de } 750 \mathrm{~cm}^{3}\right)\end{array}$ & $0,61 \mathrm{~b}$ & $28,13 \mathrm{~b}$ \\
\hline 6 & $\begin{array}{l}\text { Bolsa polietileno negro de } 17 \times 23 \\
\mathrm{~cm}\left(\text { volumen de } 1.700 \mathrm{~cm}^{3}\right)\end{array}$ & $0,70 \mathrm{ab}$ & $39,06 \mathrm{c}$ \\
\hline
\end{tabular}

Promedios con letras distintas indican diferencia significativa según la prueba Tukey $(P \leq 0,01)$. 


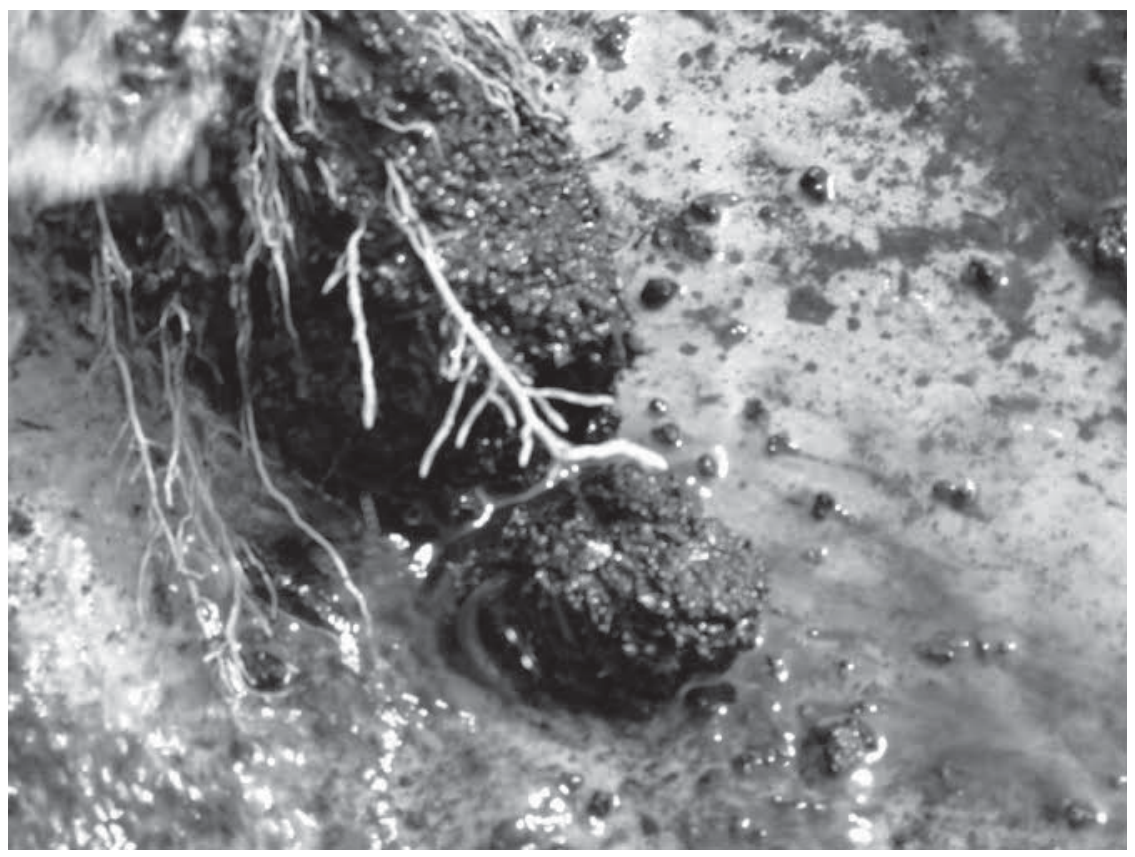

Figura 2. Deformación en la raíz pivotante en café (C. arabica L. cv. Castillo) en etapa de vivero, a los 112 ddt. Foto: L.J. Díaz M.

\section{CONCLUSIONES}

Las plántulas de café, sembradas en el contenedor de mayor profundidad $(21,8 \mathrm{~cm})$ y la bolsa de polietileno de $17 \times 23 \mathrm{~cm}$, presentaron mayor longitud de la raíz pivotante debido a una menor restricción del espacio de crecimiento y un mejor aprovechamiento del recurso hídrico.

El volumen de raíces de las plántulas de café respondió de manera directamente proporcional con la profundidad del contenedor, presentando los menores valores aquellas sembradas en bolsa de polietileno de $11 \times 20 \mathrm{~cm}$ y bandeja plástica de $17,3 \mathrm{~cm}$ de profundidad.

La mayor acumulación de biomasa en la raíz se presentó en las plántulas de café sembradas en las bandejas de mayor profundidad debido a una mejor utilización de los recursos esenciales para su crecimiento.

Las plántulas sembradas en bolsas de polietileno de $17 \times 23 \mathrm{~cm}$ mostraron los más altos porcentajes de deformación de la raíz pivotante, por lo cual no se recomienda su utilización en fase de vivero por largos períodos de tiempo.

Las plántulas sembradas en bandejas de 21,8 $\mathrm{cm}$ de profundidad presentaron los mejores resultados para las variables de crecimiento radicales determinadas en este estudio, por lo que se puede recomendar su uso para la producción comercial de café en etapa de vivero, además de disminuir la contaminación ambiental por bolsas de polietileno ya que son reutilizables por largos períodos de tiempo. 


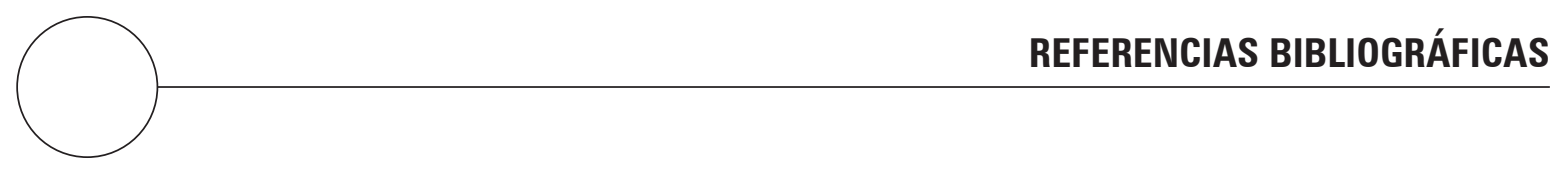

Allison, C.J., Jr. 1974. Design considerations for the RL single cell system. pp. 233-236. En: Proc. North American Containerized Forest Tree Seedling Symposium, 26-29 de Agosto 1974. Denver, CO.

Alm, A., D. Olsen y M. Lacky. 1982. Comparisons after planting of jack pine grown for varying time periods in different container systems. University of Minnesota College of Forestry. Minn. For. Res. Notes 279. St. Paul, MN.

Alvarado, G., H. Posada y G. Cortina. 2005. Castillo: Nueva variedad de café con resistencia a la roya. Cenicafé. Avance Técnico 337, 1-8.

Al-Zalzaleh, H. 2009. Root and shoot growth of Acacia saligna and Eucalyptus viminalis as influenced by container geometry. Eur. J. Sci. Res. 25, 567-573.

Arcila, J. 2000. Evite errores en el manejo de almácigos de café. Cenicafé. Avance Técnico 274, 1-7.

Arcila, J. y M. Botero. 1985. Densidad máxima de semilla para los germinadores de café. pp. 32- 56. En: Informe anual de labores de la sección de fitofisiología 1984-1985. Cenicafé, Chinchiná, Colombia.

Arcila, J., F. Farfán, M. Moreno, G. Salazar y E. Hincapié. 2007. Sistemas de producción de café en Colombia. Cenicafé, Chinchiná, Colombia.

Arizaleta, M. y R. Pire. 2008. Respuesta de plántulas de cafeto al tamaño de la bolsa y fertilización con nitrógeno y fósforo en vivero. Agrociencia 42, 47-55.

Ball, J.B. 1976. Recipientes de plástico y enrollamiento de raíces. FAO Roma. Unasylva 28 (111), p. 27.

Barrett, D.J. y R.M. Gifford. 1995. Acclimation of photosynthesis and growth by cotton to elevated $\mathrm{CO}_{2}$ : interactions with severe phosphate deficiency and restricted rooting volume. Aust. J. Plant Physiol. 22, 955-963. Doi: 10.1071/PP9950955

Berenguer, J.J., M. García Lozano, I. Escobar y M.M. Téllez. 2004. Evaluación del volumen, tiempo de uso y geometría del contenedor en cultivos en fibra de coco. pp. 163-169. Acta VII Jornadas de Sustratos de la Sociedad Española de Ciencias Hortícolas (SECH), Madrid.

Bilderback, T.E. 2001. Environmentally compatible container plant production practices. Acta Hortic. 548, 311-318. Doi: 10.17660/ActaHortic.2001.548.35
Bilderback, T.E. y W.C. Fonteno. 1991. Effects of container geometry and media physical properties on air and water volumes in containers. J. Environ. Hort. 5, 180-182.

Brandão, A.D. y L. Sodek. 2009. Nitrate uptake and metabolism by roots of soybean plants under oxygen deficiency. Braz. J. Plant Physiol. 21(1), 13-23. Doi: 10.1590/S1677-04202009000100003

Brombini, A. y P. Mazzafera. 2013. Aquaporins and the control of the water status in coffee plants. Theor. Exp. Plant Physiol. 25(2), 79-93. Doi: 10.1590/ S2197-00252013000200001

Burdett, A. 1979. Juvenile instability in planted pines. Irish Forestry 36(1), 36-47.

Carelli, M.L. y J.I., Fahl. 2006. Partitioning of nitrate reductase activity in Coffea arabica L. and its relation to carbon assimilation under different irradiance regimes. Braz. J. Plant Physiol. 18(3), 397-406. Doi: 10.1590/S1677-04202006000300006

Chirino, E., A. Vilagrosa, E. Hernández, A. Matos, A. y V. Vallejo. 2008. Effects of a deep container on morpho-functional characteristics and root colonization in Quercus suber L. seedlings for reforestation in Mediterranean climate. Forest Ecol. Manage. 256, 779-785. Doi: 10.1016/j.foreco.2008.05.035

Coopman, R. 2000. Efecto del tipo de contenedor en las porosidades del sustrato, variables morfofisiológicas y aspectos de manejo en vivero, de plantas de Pinus radiata D. Don propagadas a partir de estacas. p. 102. En: Memoria de Título. Facultad de Ciencias Forestales, Universidad de Concepción, Concepción, Chile.

Dumroese, R.K., T. Luna y T.D. Landis (eds.). 2009. Nursery manual for native plants: A guide for tribal nurseries. p. 302. En: Nursery management. Vol. 1. Agriculture Handbook 730. USDA, Washington, D.C.

Escobar, R. 2007. Eucalyptus globulus a raíz cubierta. p. 229. En: Manual de viverización. Trama Impresores, Hualpén, Chile.

Fedecafé. 2010. Historia del café. Federación Nacional de cafeteros de Colombia, Chinchiná, Colombia.

Furuta, T. 1978. Environmental plant production and marketing. Cox Publishing Company, Arcadia, CA. 
Gaitán, A., C. Villegas, C. Rivillas, E. Hincapié y J. Arcila. 2011. Almácigos de café: calidad fitosanitaria, manejo y siembra en el campo. Cenicafé. Avance Técnico 404, 1-7.

Gómez, G.L., R.A. Caballero y R. Baldión. 1991. Ecotopos cafeteros de Colombia. Federación Nacional de Cafeteros de Colombia, Bogotá.

Gutiérrez, M., R. Gómez y N. Rodríguez N. 2011. Comportamiento del crecimiento de plántulas de cacao (Theobroma cacao L.), en vivero, sembradas en diferentes volúmenes de sustrato. Corpoica Cienc. Tecnol. Agropecu. 12(1), 33-42.

Heller, H., A. Bar-Tal, S. Assouline, K. Narkis, S. Suryano, A. de la Forge, M. Barak, H. Alon, M. Bruner, S. Cohen y D. Tsohar. 2015. The effects of container geometry on water and heat regimes in soilless culture: lettuce as case study. Irrigation Sci. 33(1), 53-65. Doi: 10.1007/s00271-014-0448-y

Kafkafi, U. 2008. Functions of the root system. pp. 1340. En: Raviv, M. y J.H. Lieth (eds.). Soilless culture: theory and practice). Elsevier, Amsterdam, The Netherlands.

Kinghorn, J.M. 1974. Principles and concepts in container planting. En: Tinus, R.W., W.I. Stein y W.E. Balmer (eds.). Proc. North American Containerized Forest Tree Seedling Symposium. Great Plains Agricultural Council, Denver, Colorado, pp. 8-18.

Landis, T.D., R.W. Tinus, S.E. McDonald y J.P. Barnett. 1990. Containers and growing Media. Container Tree Nursery Manual. Vol. 2. USDA ForestService, Washington D.C.

Ledent, J.F. 2002. Déficit hídricoy crecimiento delas plantas: Respuestas al déficit hídrico: Comportamiento morfofisiológico / Modelado del crecimiento de las plantas: Manual. pp. 15-20. Fundacion Proinpa; Centro Internacional de la Papa (CIP), Lima.

Ouma, G. 2006. Growth responses of rough lemon (Citrus limon L.) rootstock seedlings to different container sizes and nitrogen levels. Agricultural Tropica et Subtropica 39(3), 182- 188.

Passioura, J.B. 2002. Soil conditions and plant growth. Plant Cell Environ 25(2), 311-318. Doi: 10.1046/j.0016-8025.2001.00802.x

Pereira, L., A.S. Dalla Côrt São Miguel, E.M. Bonfim-Silva, E. Damacena de Souza y F.D. da Silva. 2015. Influence of soil bulk density on shoot and root traits of crotalaria. Pesqui. Agropecu. Trop. 45(4), 464-472. Doi: 10.1590/198340632015v4538107
Peterson, T.A, M.D. Reinsel y D.T. Krizek. 1991a. Tomato (Lycopersicon esculentum Mill cv. 'Better Bush') plant response to root restriction. J. Exp. Bot. 42, 1233-1240. Doi: 10.1093/jxb/42.10.1233

Peterson, T.A, M.D. Reinsel y D.T. Krizek. 1991b. Tomato (Lycopersicon esculentum Mill cv. Better Bush) plant response to root restriction. II. Root respiration and ethylene generation. J. Exp. Bot. 42, 12411249. Doi: $10.1093 / j x b / 42.10 .1241$

Raviv, M., J.H. Lieth, A. Bar-Taly A. Silber. 2008. Growing plants in soilless culture: operational conclusions. pp. 545-572. En: Raviv, M. y J.H. Lieth (eds.). Soilless culture: Theory and practice. Elsevier, Amsterdam, The Netherlands. Doi: 10.1016/ B978-044452975-6.50015-0

Salazar-Arias, J. 1991. Efecto del tamaño de bolsa sobre el desarrollo de "colinos" de café. Avances Técnicos Cenicafé 170, 1-4.

Salazar-Arias, J. 1996. Efecto del tamaño de la bolsa del almácigo sobre la producción de café. Cenicafé 47(3), 115-120.

Serrada, R. 2000. Apuntes de repoblaciones forestales. Fucovasa, Madrid. pp. 18-22.

Slatyer, R.O. 1967. Plant-water relationships. Academic Press, London.

South, D.B., S.W. Harris, J.P. Barnett, M.J. Hainds y D.H. Gjerstad. 2005. Effect of container type and seedling size on survival and early height growth of Pinuspalustris seedlings in Alabama, USA. Forest Ecol. Manage. 204(2-3), 385-398.

Spomer, L. 1982. The effect of container soil volume on plant growth. HortScience 17(4), 680-681.

Tocancipá-Falla, J. 2010. Cafés in Colombia: socio-political and cultural forms of representation at the turn of the century. Int. Soc. Sci. J. 61(202), 425-436. Doi: 10.1111/j.1468-2451.2011.01770.x

Vilanova, C., A., Iglesias y M. Porcar. 2015. The coffeemachine bacteriome: biodiversity and colonisation of the wasted coffee tray leach. Nature Scientific Reports 5, 17163. Doi: 10.1038/srep17163

Wallach, R. y M. Raviv. 2005. The dependence of moisture-tension relationship and water availability on irrigation frequency in containerized growing media. Acta Hortic. 697, 293-300. Doi: 10.17660/ ActaHortic.2005.697.36

Weston, L.A. y B.H. Zandstra. 1986. Effect of root container size and location of production on growth and yield of tomato transplants. J. Amer. Soc. Hort. Sci. 111(4), 498-501. 
Wightman, K.E. 2000. Prácticas adecuadas para los viveros forestales. Guía Práctica para los viveros comunitarios. Centro Internacional para Investigación en Agroforestería (ICRAF), Nairobi, Kenia.

Xu, G., S. Wolf y U. Kafkafi. 2001. Interactive effect of nutrient concentration and container volumen on flowering, fruiting and nutrient uptake of sweet pepper. J. Plant Nutr 24(3), 479-501. Doi: 10.1081/ PLN-100104974

Zaharah, S. e I.Razi, 2009. Growth, stomata aperture, biochemical changes and branch anatomy in mango (Mangifera indica) cv. Chokanan in response to root restriction and water stress. Sci. Hortic. 123(1), 58-67. Doi: 10.1016/j.scienta.2009.07.022 